Erschienen in: Kämper, Heidrun/Eichinger, Ludwig M. (Hrsg.): Sprache -

Kognition - Kultur. Sprache zwischen mentaler Struktur und kultureller Prägung. - Berlin, New York: de Gruyter, 2008. S. 14-23. (Institut für Deutsche Sprache. Jahrbuch 2007), https://doi.org/10.1515/9783110970555-003

Klaus P. Hansen

\title{
Sprache und Kollektiv
}

\author{
Ein Essay
}

\begin{abstract}
Sprache ist kein natürliches Phänomen, sondern ein kulturelles. Sie entwickelte sich nicht als Konsequenz biologischer Voraussetzungen, entstand vielmehr, sozusagen spontan, aus den Kommunikationsbedürfnissen menschlicher Kollektivität. Wo immer der homo sapiens sich zusammenrottete, schuf er Verständigungsmöglichkeiten, sodass es die Sprache nicht gibt, sondern nur Sprachen. Da sie kulturelle Phänomene sind, lassen sie sich mit den Begriffen der Kulturwissenschaft oft angemessener beschreiben als mit denen der Linguistik.
\end{abstract}

Das Walten der Natur, davon ist die Mehrheit der Biologen überzeugt, lässt sich durch das darwinistische Paradigma angemessen erklären. Obwohl auch der Mensch Teil der Natur ist, stößt es bei ihm an eine Grenze. Darwin setzte eine Grundspannung an, aus der eine nie erlahmende und universelle Energie floss, die zwischen Gattung bzw. Art auf der einen und der jeweiligen Umwelt auf der anderen Seite. Diejenigen Gattungen überlebten, deren Ausstattung an die Umwelt angepasst werden konnte; die andern, denen es nicht gelang, sich im vorgegebenen Lebensraum einzurichten, gingen unter. Dieser Test des Sich-Arrangierens wurde von den Menschenaffen bestanden ebenso wie vom homo sapiens, der sich aufgrund genetischer Mutation, also sozusagen als Fehlgeburt, aus ihnen entwickelt hatte. Allerdings wurde bei dieser letzten Gattung die Testanordnung verändert. Die Neuentwicklung homo sapiens verdiente ihren Namen insofern, als sie den Spieß herumdrehte und nicht die Gattung der Umwelt anpasste, sondern die Umwelt der Gattung. Die Idee eines Arrangements, das Darwin zum Garant des Überlebens erhob, blieb dabei gewahrt, nur dass auf den Kopf gestellt wurde, wer sich wem anpasste. Durch Ackerbau und Viehzucht griff der Mensch in das natürliche Wachstum ein und löste sich so vom zufälligen Nahrungsangebot der Umwelt. Er machte sich, wie die Bibel es später formulierte, die Erde untertan und trat damit aus den Bedingungen der Natur heraus, was auch bedeutet, dass er mit Darwins Paradigma nicht zu fassen ist. Die Gattung Mensch emanzipierte sich von der Natur und schwang sich vom Untertan zum Herrscher auf.

Wie weit diese Emanzipation von der Natur ging, bzw. wie weit sie gehen darf, diskutieren wir gerade wieder. Die Jahrhunderte lang glänzend funktio- 
nierende Manipulation des Gegebenen, die allerdings schmutzige Spuren in der Atmosphäre hinterließ, stößt anscheinend an eine Grenze. Was aber keinen Rückfall in den Naturzustand auslösen und Darwin einen erneuten Triumph bescheren wird: Wir werden Rousseaus Aufforderung nicht befolgen und der Zivilisation abschwören, sondern wir werden die unsauberen Techniken durch saubere ersetzen. Der Herrscher wird eher untergehen, als dass er wieder zum Untertan wird.

Was waren die Bedingungen der Emanzipation von der Natur? Es sind zwei, die zusammengehören: Kollektivität und, um sie zu ermöglichen, Kommunikation. Was zunächst nicht einleuchten mag, da auch im Tierreich beides vorzukommen scheint. Wölfe leben in Rudeln, Bienen in Völkern, und Ameisen bilden vielleicht sogar einen Staat. Kollektivität, sei sie instinktiv oder nicht, setzt Kommunikation voraus. Wölfe verständigen sich durch Laute und Verhalten; Bienen kommunizieren, wie man inzwischen weiß, durch Flugbewegungen. Sie tanzen eine Art geographische Skizze, um den Artgenossen die Lage von Futterquellen mitzuteilen. Bienen und Ameisen befinden sich daher auf der Schwelle zur Emanzipation, ohne sie aber zu überschreiten: Zwar leben sie in komplexen Kollektiven, in denen Wissen durch Kommunikation weitergegeben wird, aber diese Existenzformen bleiben auf das Kreatürliche, d.h. auf das Überleben und die Erhaltung der Art beschränkt.

Nur der Mensch steigerte die Emanzipation bis zur Herrschaft über die Natur. Auch die menschliche Gattung lebt in komplexen Kollektivverbänden; auch sie gibt Wissen durch Kommunikation weiter und betreibt, was Bienen und Ameisen auch tun, Vorratshaltung im großen Stil. Aber der Mensch überschreitet die Schwelle insofern, als sein Trachten nicht nur dem Überleben dient. Selbst von diesem fundamentalsten Axiom der Natur befreite er sich. Sobald Menschen Altäre errichten, Rituale zelebrieren und Symbole in Höhlenwände oder Totempfähle ritzen, betreiben sie Wirklichkeitsdeutung und setzen sich eigene Ziele. An die Stelle der Erhaltung der Art tritt im Kollektiv geschaffener Sinn.

Auch diese letzte und endgültige Emanzipation lässt sich gerade heute eindrucksvoll studieren. Selbstmordattentäter sind Menschen, die, um einen bestimmten kollektiven Sinn zu befördern, ihr Leben vernichten. Um ihren Gottesstaat zu errichten, setzen sie sich über das zentrale Prinzip der Natur, die Erhaltung des Lebens, hinweg. Die Existenz von Märtyrern und Selbstmordattentätern, die sich beide in Kollektiven aufgehoben wissen, müsste den letzten Zweifler davon überzeugen, dass menschliche Existenz nicht darwinistisch erklärt werden kann, sondern nur kollektivistisch und kulturell.

Der Natur liegt nur die Art oder Gattung am Herzen und somit auch das einzelne Lebewesen als deren Baustein. Indem es überlebt und seine Erbinformation weitergibt, ist der Fortbestand der Gattung gewahrt. Die Emanzipation von der Natur bricht mit diesem Gattungsbezug. An seine Stelle tritt das Kollektiv. Eine Untereinheit der Gattung übernimmt mithin die Funktion 
der Befehlszentrale. Jetzt regieren nicht mehr nur die gattungstypischen Instinkte, deren Leitfunktion auf den Bereich des Kreatürlichen beschränkt werden, sondern es kommen die Maximen des Kollektivs hinzu, die sich gegen die Instinkte durchsetzen können. Sie bestimmen Verhalten wie Kommunikation und legen fest, was richtig und vernünftig ist. Die Art oder Gattung teilt sich in Kollektive auf, und wie gewichtig dieser Schritt für die Emanzipation von der Natur ist, erkennen wir daran, dass sich artgleiche Kollektive in kleinen und großen Kriegen bekämpfen, die dem Axiom der Erhaltung der Art zuwider laufen.

Im Unterschied zu den Arten lässt sich die Entstehung von Kollektiven nicht darwinistisch durch Mutation des Erbmaterials erklären. Kollektive sind keine genetischen Zufallsprodukte, sondern solche eigenständiger Gestaltung. Sie bündeln individuelle Interessen und unterwerfen sich der AutoPoiesis Luhmanns. Was das Überleben betrifft, müssen sich die Kollektive allerdings ebenfalls in ihrer Umwelt bewähren. Aber das Maß aller Dinge ist es nicht. Wichtiger ist vielmehr der kollektive Sinn, der, das zeigte der Hinweis auf Märtyrer und Selbstmordattentäter, stärker sein kann als der Lebenswille.

Kollektiver Sinn setzt sich aus Einheiten des Gleichverhaltens zusammen. In der Gruppe der islamistischen Selbstmordattentäter wäre ein solches Gleichverhalten das Freitagsgebet. Gleichverhalten kann auf zweierlei Weise konstituiert werden, entweder durch Reglementierung, also durch Druck, oder aufgrund freiwilliger, zum Teil unbewusster Übereinkunft. Gegenüber politischen Reglementierungen, die auf kontrollierten Normen und Gesetzen beruhen, sind die kulturellen weniger geplant als vielmehr spontan entstanden. Wir wollen sie mit dem kulturwissenschaftlichen Begriff Standardisierung bezeichnen. Da wir über Sprache reden, die nur geringfügig reglementiert ist, interessieren uns eben diese kulturellen Standardisierungen.

Kollektive sind ohne Kommunikation nicht denkbar. Kollektivität wird durch Kommunikation konstituiert. Deshalb stehen die Standardisierungen der Kommunikation als Bedingung von Kollektivität an erster Stelle. Indem der Einzelne mit den anderen der Gruppe kommuniziert, wird er Teil des Kollektivs. Kollektive Kommunikation ist etwas anderes als natürliche Kommunikation. Diese Unterscheidung lässt sich anhand der Kriterien Herkunft und Ziel treffen: Der die Artgenossen warnende Angstschrei des Schimpansen stammt aus dem gattungseigenen Kommunikations-Repertoire und dient dem Ziel des Überlebens. Jenseits dieser natürlichen Kommunikation liegt der Satz eines deutschen Sprechers, der sagt: „Einsteins Relativitätstheorie wirft mehr Fragen auf, als sie beantwortet." Dieser Satz stammt nicht aus dem Kommunikations-Repertoire der Gattung - denn ein Franzose oder ein deutscher Arbeiter versteht ihn nicht - und er dient nicht dem kreatürlichen Überleben. Der Satz stammt aus einem Kollektiv, dem der deutschen Physiker. Er dient keinesfalls dem Überleben, wahrscheinlich noch nicht einmal dem beruflichen. Es handelt sich mithin um eine Kommunikation, die weit über natürlich Gegebenes hinausgeht. 
Sprache ist ein kollektives Produkt. Nicht die Natur ließ es entstehen, sondern es geht zurück auf spontane Impulse, die, einmal angestoßen, eine Art organisches Wachstum entfalteten. Stünde die Natur Pate, wäre es eine Sprache für die gesamte Gattung Mensch; eine solche Sprache aber gibt es nicht. Die Sprachen, die wir kennen, sind Kollektiv-Sprachen. Sie entstanden in den verschiedenen Urhorden des homo sapiens, die in räumlicher Trennung an verschiedenen Punkten der Erde vegetierten.

Dass diese verschiedenen Sprachen gewisse Gemeinsamkeiten zeigen, tut der These von der kollektiven Entstehung keinen Abbruch. Dass sie alle Vokal- und keine Gesten- oder Bewegungssprachen sind, hat pragmatische Gründe. Die Kommunikation per Geräusch hat den Vorteil, dass man nicht die Hände frei haben und mit dem Adressaten keinen Blickkontakt aufnehmen muss. Die Geräuschsprache lässt sich außerdem leichter verschriftlichen, also haltbar machen. Ohr und Auge sind unsere komplexesten Sinnesorgane, und daher verwundert es nicht, dass sie zu Kommunikationsrezeptoren wurden. Dass alle Sprachen Subjekt und Objekt unterscheiden und den Modus Handlung ausdrücken können, ist auch kein Argument für die natürliche und gemeinsame Herkunft. Subjekt, Objekt und Handlung sind Kategorien der Wirklichkeit, und da sich Sprache auf Wirklichkeit bezieht, muss sie diese Kategorien abbilden.

Sprache funktioniert über Zeichen. Einem Bedeutungsträger wird eine Bedeutung zugeordnet. Diese Zuordnung, das ist die große, immer noch nicht genügend gewürdigte Entdeckung von Ferdinand de Saussure, ist willkürlich. Das hat zur Folge, dass Sprache nur kollektiv auf der Grundlage von Verabredung funktioniert. Dass das deutsche Wort Wolke diese weiße Masse am Himmel bedeutet, kann außerhalb Deutschlands niemand ahnen. Die Bedeutung muss vielmehr verabredet bzw. gelernt sein. Verabredungen aber sind Sache der Kultur. Verabredungen trifft man in Kollektiven und nicht innerhalb von Arten.

Wie haben wir uns die Entstehung solcher Verabredungen vorzustellen? Nehmen wir an, ein Flughafenbus, der Passagiere zu ihrer Maschine bringt, wird überfallen und die Insassen als Geiseln genommen. Zufällig spricht keiner die Sprache des anderen und keiner ist des Englischen mächtig. Damit liegt die theoretische Ursituation der Kollektivbildung vor: Weil man räumlich zusammen und aufeinander angewiesen ist, braucht man Kommunikation, die aber nicht existiert. Obwohl keiner die Sprache des anderen spricht, werden sich jedoch, vorausgesetzt die Gruppe bleibt länger zusammen, Kommunikationsmittel ergeben.

Das Hochheben des Eimers, in dem man Wasser holt, wird die Bedeutung trinken bekommen. Wenn es nur einen Eimer gibt, wird bald das Hochheben der geschlossenen Hand genügen, um die Bedeutung abzurufen. Das ursprünglich analoge Zeichen wird sich immer weiter digitalisieren. Da Trinken im heißen Bus positiv besetzt ist, könnte die Gebärde polysem werden und neben trinken auch Glück bedeuten. Wenn genügend Zeichen versammelt 
sind, würden Kombinationsregeln folgen, die das Repertoire an Bedeutungen erweitern. Ginge die Welt dann unter und nur diese Busladung bliebe als moderne Arche Noah übrig, würde nach einigen Generationen eine vollwertige Sprache vorhanden sein. Ihre Entstehung, das zeigt unser Beispiel, ist ohne den Rückgriff auf Natur denkbar. Ich brauche weder Gott noch ein planvolles Universum.

Gegen diese Metaphysik, die sich als Naturwissenschaft tarnt, setze ich meine These vom kollektiven Ursprung der Sprachen. Aus ihr folgt eine zweite. Die Grundlagen der Sprachen sind mit denen anderer Kollektivphänomene identisch. Anders formuliert, Sprache ist ein Stück Kultur. Das wollen bestimmte Gruppierungen der Sprachwissenschaft nicht wahrhaben, die von der Besonderheit, wenn nicht Einzigartigkeit ihres Gegenstandes überzeugt sind. Sie tun so, als sei ihm nichts vergleichbar, dabei steht er brav in einer Reihe mit anderen kollektiven bzw. kulturellen Erscheinungen. Die Sprache mag zwar viele der kulturellen Nachbarphänomene an Komplexität übertreffen, was aber keine qualitative Andersartigkeit bedeutet.

Diese zweite These lässt sich an der Herrenmode exemplifizieren. Sie bildet ein Zeichensystem, das sowohl über Semantik als auch Grammatik verfügt und letztendlich genauso funktioniert wie Sprache. Hier wie dort erkennen wir eine begrenzte Zahl von polysemen Einzelzeichen, die durch Kombinationsregeln, also eine Grammatik, unbegrenzt viele eindeutige Aussagen mitteilen können. Solche Aussagen, um sich zunächst auf die standardisierten zu beschränken, wären Festlichkeit (Smoking), Eleganz (Anzug), sportliche Eleganz (Kombination), Freizeit oder Zwanglosigkeit (Jeans, Pullover, Lederjacke) und Sportausübung (Jogginganzug). Den Wortarten vergleichbar, lassen sich die Einzelzeichen bestimmten Kategorien zuordnen, nämlich Form, Farbe und Material, die - wie die Wortarten in einem Satz - immer zusammen auftreten. Die Polysemie der Einzelzeichen ist in allen Kategorien unterschiedlich stark. Die Form Jackett bedeutet je nach Zeichenverknüpfung Festlichkeit (Smoking), Eleganz (Anzug) oder Sportlichkeit (Kombination). Ebenso vieldeutig ist die Farbe Schwarz. Sie verkündet neben Festlichkeit auch Trauer und im Sonderfall der Motorradkleidung, die meist aus schwarzem Leder besteht, verweist sie auf einen speziellen Kontext. Die Farbe Rot ist demgegenüber weniger bedeutungsoffen wie auch das Material Seide, das im Unterschied zur Schurwolle, die sich für viele Anlässe eignet, auf Festlichkeit und Formalität festgelegt ist. Eine ähnliche Unsystematik der Polysemie lässt sich bei den Einzelzeichen der Sprache, den Lexemen erkennen. Nicht alle sind vieldeutig und, wenn sie es sind, werden durch den Kontext die nicht gewünschten Bedeutungen eliminiert.

Den Regeln der Grammatik vergleichbar, sind die Verknüpfungen der textilen Einzelzeichen standardisiert. Um die Aussage Eleganz zu übermitteln, müssen Jackett und Hose aus gleichem und gleichfarbigem Material sein; in Art einer Redundanz wird durch Oberhemd und Krawatte der Eindruck verstärkt. Durch Modifikation des Materials und der Farbe sind Variationen 
der Aussage möglich: Ein Kordanzug ist weniger elegant als einer aus Schurwolle, insbesondere wenn ihn eine mutige Farbe darin unterstützt. Mit leicht geänderter Verknüpfung und weiteren Accessoires kann der Anzug zum Smoking aufgerüstet werden. Schwarz ist für ihn Pflicht, und das Revers des Jacketts muss mit Seide überzogen sein; an die Stelle der Krawatte tritt die Fliege, und das Oberhemd darf neben dem Stehkragen feminine Verzierungen aufweisen; des weiteren sollten Lackschuhe, egal wie unbequem sie sind, das Bild abrunden. Bei diesem Aufzug ist die Redundanz der Charakterisierung besonders groB. Die Aussage Festlichkeit kann aber auch durch eine Zeichenfolge hervorgerufen werden, die das Prinzip Anzug, also Gleichheit von Hose und Jacke, empfindlich stört. Zu einer immer noch schwarzen Hose kann ich ein weißes oder cremiges dinner-jacket anziehen und erziele dieselbe Wirkung. Genau wie bei der Sprache gibt es bei der Herrenmode mithin Synonyme.

Betrachten wir noch eine weitere Besonderheit. Auch die uneleganteste Bekleidung, der Jogginganzug, ist nach der Zeichenverknüpfung des vornehmeren Bruders Anzug kombiniert, aber eben nicht komplett. Jacke wie Hose sind zwar aus gleichem Stoff, aber an die Stelle des Jacketts tritt eine saloppe Jacke. Der Schnitt ist außerdem weit und das Material synthetisch. Trotz der geringen Abweichungen wird so das absolute Gegenteil von festlich erreicht. Wir sehen: Mit Hilfe weniger, meist polysemer Zeichen können über Kombinationen viele Botschaften formuliert werden, wobei es nicht nur eine Möglichkeit gibt, sondern mehrere.

Die Herrenmode bildet genauso ein komplexes und unsystematisches System wie die Sprache. Erweitern wir den kleinen Ausschnitt unseres Beispiels durch einen schnellen Blick auf die Jugendmode, die trotz ihres Aufbegehrens und ihres geballten Antagonismus ein logischer Teil des Bereichs Bekleidung darstellt. Bei aller modischen Variation wahrte konventionelle Kleidung zwei Prinzipien, das der Funktionalität und das der Passform. Indem sie bewusst gegen diese Prinzipien verstößt, vollzieht die Jugend einen radikalen Systemwechsel. Die Schnürsenkel werden nicht mehr gebunden, was unfunktionell, wenn nicht gefährlich ist; die Pullover sind zu weit, die Ärmel zu lang und der tiefer gelegte Schritt der Hose behindert die Fortbewegung. Der Schirm der baseball-cap, der ursprünglich vor der Sonne schützen sollte, wird zur Seite oder nach hinten gedreht.

Das System Kleidung ist komplex und in seinen Aussagen so differenziert, dass es sprachlich nur mit Mühe darstellbar ist. Betrachten wir nur die Kategorie Sportlichkeit. Von der sogenannten Kombination, d. h. Jackett und Hose aus verschiedenen Materialien, über Pullover mit Jeans oder Lederjacke mit Cordhose bis zum Jogging-Anzug werden Abstufungen geboten, für die uns in dieser Nuancierung die Worte fehlen. Eine weitere Ähnlichkeit zur Sprache: Das System Kleidung erlaubt und ermöglicht Kreativität. Das Konventionelle kann durch neue Kombinationen in verschiedene Richtungen gedehnt werden. Wie die Dichter die Sprache erweitern und verändern, schafft 
der Modeschöpfer mit Hilfe von moderaten Tabubrüchen Arrangements mit neuen Aussagen. Er könnte zur Smokingjacke eine schwarze Jeans entwerfen und damit eine Tradition der Gegenwart anpassen.

Man würde noch weitere Ähnlichkeiten entdecken, die sich alle in folgender Erkenntnis treffen: Das Bedeutungssystem Kleidung ist in seinen Grundlagen, seiner Komplexität und seiner Dynamik durchaus dem System Sprache vergleichbar. Dennoch käme niemand auf die Idee, unser Kleidungsverhalten auf die Natur zurückzuführen. Niemand würde den Ursprung der Grammatik der Herrenmode in unseren Genen oder Synapsen suchen. Niemand käme auf die Idee, es von seiner kollektiven Entstehung abzulösen und als Naturphänomen zu verdinglichen.

Große Teile der Sprachwissenschaft und Linguistik, so jedenfalls macht es auf Außenstehende den Eindruck, halten Sprache - vielleicht eher unbewusst als bewusst - für etwas Einzigartiges, für ein Ding, nicht an sich, aber für sich. Diese Verdinglichung, die sich wahrscheinlich vor allem aus der angeblichen Naturgegebenheit begründet, schlägt sich in aller Deutlichkeit im gerne verwendeten Begriff System nieder. Sprache wird als System gesehen. Dabei denken wir an automatisierte Regelkreise, an fest ineinandergreifende Interaktion und austarierte Eigendynamik. Das Sprachsystem wird dem Planetensystem gleichgesetzt: Beide bewegen sich eigendynamisch nach naturwissenschaftlicher Gesetzmäßigkeit.

Diese Prämisse, die immer noch nicht laut und deutlich genug reflektiert wird, schlägt sich in Vorlieben und Methoden nieder. Nachdem die Linguistik durch die Zeichentheorie groß wurde und die historische Sprachwissenschaft ablöste, stürzte sie sich in erster Linie auf die Grammatik. Sie wurde zum Forschungsgegenstand par excellence, weil man hier den Systemcharakter der Sprache am deutlichsten zu finden hoffte. Dazu musste die Tradition der lateinischen Grammatik, die nur eine Sammlung von Regeln und Ausnahmen darstellte, überwunden und durch Beschreibungsmodelle ersetzt werden, welche die Systematik stärker herausarbeiteten. Die Euphorie ist inzwischen allerdings verflogen, und im Fremdsprachenunterricht herrscht weiterhin unangefochten die lateinische Grammatik. Warum gelang die Neuorientierung nicht? Ich meine, die Prämisse war falsch. Grammatik bildet eben kein System im strengeren Sinne und ist außerdem nicht der für die Verständigung wichtigste Teil.

Nach der Diagnose komme ich jetzt zur Therapie. Viele Wissenschaften vollzogen in der letzten Zeit einen cultural turn. Ansätze dazu, so wurde ich informiert, sind auch in der Linguistik vorhanden. Sie sind aber noch zu zaghaft und wagen noch nicht die paradigmatische Kehrtwende. Sie scheuen sich noch, die traditionellen Prämissen und Beschreibungsansätze über Bord zu werfen und durch neue mit kulturwissenschaftlicher Ausrichtung zu ersetzen. Wie würde das aber aussehen, wenn die in dieser Disziplin entwickelte Begrifflichkeit auf den Gegenstand Sprache übertragen würde? Greifen wir zwei zentrale und umfassende Begriffe heraus, den der Standardisierung, den 
ich in die Debatte warf, und den des ,selbstgesponnenen Bedeutungsgewebes", der auf den großen, gerade verstorbenen amerikanischen Kultur-Anthropologen Clifford Geertz zurückgeht.

In meinem UTB-Band Kultur und Kulturwissenschaft, der bald in 4. Auflage vorliegt, definiere ich Kultur als die Summe der Standardisierungen, die in einem Kollektiv gelten. Obwohl es zum guten Ton gehört, über die Vieldeutigkeit und Uneinheitlichkeit des Kulturbegriffs zu klagen - Clyde und Kluckhohn zählten 1952 über hundert davon - gelingt es dieser Definition, alle Kulturansätze unter einen Hut zu bringen. So unterschiedliche Phänomene wie Sitten, Bräuche, Kunst, Umgangsformen, Kleidungsstile, Werte, Mythen und Weltanschauungen zählen die kulturwissenschaftlichen Disziplinen zur Kultur und bilden aus ihnen die verschiedensten Kulturbegriffe. Doch hinter der Unterschiedlichkeit der Gegenstände und der Verschiedenheit der Begriffe verbirgt sich eine Gemeinsamkeit. Phänomene wie die Sitten eines Stammes, die Kunst einer Epoche, die Umgangsformen einer Schicht, Mode oder Diskurse existieren stets kollektiv und sind als Gleichverhalten erkennbar. Ihre Erscheinungsform lässt sich als Konvention, Tradition oder Norm bezeichnen oder noch allgemeiner als kollektive Gewohnheit oder eben Standardisierung. Kultur meint das Wiederkehrende, das sich Wiederholende. Sie meint jene Regelmäßigkeiten, denen man sich freiwillig unterwirft. Kultur missachtet das Individuelle, Besondere, Originelle zugunsten des Kollektiven. Es steht zu Recht an erster Stelle, denn erst auf seinem Hintergrund wird das Individuelle sichtbar. Vor Shakespeare war es kulturelle Norm, Tragödie und Komödie zu trennen; er aber, darin Genie, vermischte beides. Er wagte als Erster „to mingle kings and clowns“, löste dadurch die herrschende Standardisierung ab und schuf eine neue.

Der Begriff Standardisierung, der sich, wie ich beobachte, immer mehr durchsetzt, passt vorzüglich auf sprachliche Phänomene. Die Einzelzeichen, die Lexeme sind insofern standardisiert, als sie verabredet sind. Ihr digitaler Charakter funktioniert aufgrund von Standardisiertheit. Grammatische Regeln sind ebenfalls Standardisierungen. Gegenüber dem Begriff Regel hat der Begriff Standardisierung aber den Vorteil, dass man keine Ausnahmen einführen muss, um die Regel zu retten. Denn auch Ausnahmen sind Standardisierungen. Im Unterschied zu den alten und neuen Grammatikterminologien suggeriert Standardisierung nicht das Prinzip der Regel oder der Norm, so als wäre ein sprachlicher Gesetzgeber am Werk, sondern rekurriert auf Übereinkünfte eines Kollektivs.

Doch der Begriff Standardisierung ist auch jenseits der Grammatik sinnvoll. Idiome, Redewendungen und alles, was unter Sprachgebrauch oder Kontextabhängigkeit fällt, ist Standardisierung. In deutschen Parks lesen wir Schilder mit der Aufschrift „Rasen nicht betreten“. In allen Parks von Flensburg bis Passau finden wir nur diesen Text, und nicht etwa - genauso verständlich - „,halten Sie sich vom Rasen fern“. In England lautet die entsprechende Standardisierung „keep off the gras“. Die Variante „don't step on 
the lawn" würde zwar verstanden, aber als ungewöhnlich empfunden. Ziel der Sprache ist also nicht nur Verständlichkeit, sondern auch die Produktion von Vertrautheit, die das Kollektiv- und Identitätsgefühl stets aufs Neue bestätigt. Mit Hilfe des Begriffs Standardisierung ließe sich der Primat der Grammatik überwinden, der bis tief in den Fremdsprachenunterricht reicht. Aufgrund dieses Primats misst der Sprachenlehrer mit zweierlei Maß. Ein grammatischer Verstoß wird als Fehler angestrichen, während der Verstoß gegen den Sprachgebrauch nur unterkringelt wird. Dabei sind beide gleichwertige Standardisierungsbrüche. Entweder muss beides als Fehler gelten, oder aber es werden nur solche Verfehlungen geahndet, welche die Verständigung behindern. Textsorten, Sprechakte, Stile, Rhetorik, alles das sind Standardisierungen. Insofern würde mit Hilfe dieses kulturwissenschaftlichen Begriffs der Zusammenhang der verschiedenen Bereiche und Aspekte der Sprache sichtbar (Semantik, Grammatik, Pragmatik), und der Grammatikwahn verlöre an Boden.

Zu meinem zweiten Begriff: Clifford Geertz, der die Renaissance des Kulturbegriffs 1973 mit seinem Buch The Interpretation of Cultures (deutsch: Dichte Beschreibung 1983) auslöste, bezeichnet Kultur als ,selbstgesponnenes Bedeutungsgewebe“. Dieser Begriff erscheint mir ebenfalls hilfreich, um das Phänomen Sprache zu fassen. Gehen wir die einzelnen Facetten dieser berühmt gewordenen Prägung durch, die anders ansetzt als der Begriff Standardisierung.

Dass Sprache Bedeutungen übermittelt, ist selbstverständlich. Neu ist aber, dass alle kulturellen Phänomene als Bedeutungsträger erkannt werden. Dass Kleidung nicht nur wärmt, sondern eine Bedeutung übermittelt, haben wir besprochen. Das Gleiche tun Bräuche, Rituale, aber auch Alltagsgegenstände wie Autos, Wohnungseinrichtungen, Architektur. Wir leben in einer bedeutungsgetränkten Welt, und die moderne life-style Generation schafft sich ihre multiplen Identitäten durch Botschaften sendende Konsumgüter. Der Mercedes übermittelt die Botschaft Solidität; der BMW Sportlichkeit; der Sprit-fressende und $\mathrm{CO}_{2}$ ausstoßende Off-Roader inszeniert seinen Fahrer als Naturburschen, der das Abenteuer sucht etc. Geertz ist Kultursemiotiker, und kulturelle Phänomene haben für ihn Zeichencharakter. Dieser Ansatz geht zurück auf den französischen Linguisten Roland Barthes, den Geertz allerdings nicht zur Kenntnis nimmt.

Das Bedeutungsgewebe ist „selbstgesponnen “. Das Wörtchen selbst meint nicht die Individuen, sondern die Kollektive. Kulturelle Phänomene sind keine Naturgegebenheiten, sondern kollektives Menschenwerk. Sie entstehen vielleicht durch individuelle Initiative, müssen sich aber im Kollektiv bewähren. Ein Markenprodukt, nehmen wir wieder das Beispiel Mercedes, wird von einem Team aus Individuen entworfen und durch das größere Kollektiv Markt akzeptiert. Durch Qualität, Design, Werbung gelang es, das Produkt mit den Suggestionen Verlässlichkeit, Sicherheit und Status aufzuladen. Den ersten Schritt tat also Mercedes selbst, der ohne eine Annahme durch das Kol- 
lektiv der Autofahrer in einer Sackgasse gelandet wäre. Diese selbstgesponnenen Suggestionen sind das wertvollste an einem Mercedes, und nur für sie zahlen wir den hohen Preis.

Was meint Geertz mit ,gesponnen“? Sicherlich passt dieses Wort zu Gewebe, aber das ist nicht der einzige Grund für seine Wahl. Kulturelle Phänomene werden nicht geplant; sie werden auch nicht durch einen Geistesblitz ins Leben befördert. Vielmehr, so lautet eine andere Metapher, wachsen sie organisch. Auch Sprachen werden selbst gesponnen und wachsen organisch. Genau genommen wissen die Kulturwissenschaftler nicht, was organisches Wachstum heißt. Sie wissen nur, dass der Entstehungsprozess irgendwo zwischen absichtsvoller Planung und zufälliger Spontaneität anzusiedeln ist.

Warum Gewebe? Geertz formuliert hier eine Alternative zum Begriff System. Dieser enthält Regelmäßigkeit, Austariertheit, Funktionalität und Geschlossenheit als Merkmale. Jener aber kommt weniger mathematisch, geplant und reglementiert daher. Gewebe suggeriert Zusammenhang und betont, dass alle Teile miteinander funktionieren. Alles ist vernetzt; überall bestehen Bezüge oder können neue eröffnet werden. Ich zeigte das an der Jugendmode, die trotz ihrer Andersartigkeit in die Bezüge des Gewebes gehört. Ein Gewebe ist nicht rund und abgeschlossen; an ihm kann und wird jeder Zeit weiter gesponnen. Ohne Abstriche erfasst dieser Begriff das Phänomen Sprache: Auch sie ist ein kollektiv geknüpftes Bedeutungsgewebe.

Ich würde gerne noch eine dritte These vortragen, wozu die Zeit aber nicht reicht. Diese These würde auf meinem Ansatz der Relation von Sprache und Kollektiv aufbauen und zu beweisen versuchen, dass die Art der Sprache je nach Art des Kollektivs variiert. Fachsprachen, Dialekte und Soziolekte dienen anderen Kollektivarten als Hochsprachen, die vor allem eine nationale, öffentliche Kommunikation sicherstellen. Deshalb werden sie auch staatlich überwacht, wohingegen Schichten- und Regionalsprachen völlig sich selbst überlassen sind. Das hätte meine These von der kollektiven Herkunft der Sprache abrunden können. 